\section{Jung Hyun Park, Jong Yeol Kim}

Department of Neurosurgery, Kosin University Gospel Hospital, Kosin University College of Medicine, Busan, Korea

\section{Corresponding Author:}

Jong Yeol Kim

Department of Neurosurgery, Kosin University Gospel Hospital, Kosin University College of Medicine, 262 Gamcheon-ro, Seo-gu, Busan 49267, Korea

Tel: +82-51-990-6465

Fax: +82-51-990-3042

E-mail: spinekjy0716@gmail.com

Received: April 24, 2017

Revised: December 7, 2017

Accepted: December 8, 2017

\title{
latrogenic Spinal Subarachnoid Hematoma after Diagnostic Lumbar Puncture
}

Spinal subarachnoid hematoma (SSH) following diagnostic lumbar puncture is very rare. Generally, $\mathrm{SSH}$ is more likely to occur when the patient has coagulopathy or is undergoing anticoagulant therapy. Unlike the usual complications, such as headache, dizziness, and back pain at the needle puncture site, SSH may result in permanent neurologic deficits if not properly treated within a short period of time. An otherwise healthy 43-year-old female with no predisposing factors presented with fever and headache. Diagnostic lumbar puncture was performed under suspicion of acute meningitis. Lumbar magnetic resonance imaging was performed due to hypoesthesia below the level of T10 that rapidly progressed after the lumbar puncture. SSH was diagnosed, and high-dose steroid therapy was started. Her neurological symptoms rapidly deteriorated after 12 hours despite the steroids, necessitating emergent decompressive laminectomy and hematoma removal. The patient's condition improved after the surgery from a preoperative motor score of $1 / 5$ in the right leg and $4 / 5$ in the left leg to brace-free ambulation (motor grade 5/5) 3-month postoperative. The patient was discharged with no neurologic deficits. Critical complications such as SSH can be fatal. Therefore, a patient undergoing lumbar puncture must be carefully observed. A hematoma that convincingly compresses the spinal cord or cauda equina on imaging results requires early surgical decompression and hematoma removal.

Key Words: Lumbar puncture, Spinal subarachnoid hematoma, Spinal cord injury, Spinal hematoma

\section{INTRODUCTION}

Patients may suffer from various complications after diagnostic lumbar puncture, including headache, dizziness, and pain at the needle puncture site ${ }^{9,13)}$. One of the most critical among these is spinal subarachnoid hematoma (SSH). SSH is generally iatrogenic, but it may develop due to trauma or comorbidities such as coagulopathy. Despite surgical decompression and active rehabilitation treatment, SSH may cause permanent neurologic deficits ${ }^{10,11,14)}$. Here we report a patient who suffered iatrogenic SSH with delayed paraplegia, which developed following diagnostic lumbar puncture.

\section{CASE REPORT}

\section{History and Presentation}

Spinal Neurosurgery Society

This is an open access article distributed under the terms of the Creative Commons Attribution Non-Commercial License (http://creativecommons.org/licenses/bync/4.0/) which permits unrestricted noncommercial use, distribution, and reproduction in any medium, provided the original work is properly cited.
A 43-year-old female patient visited the Emergency Department of our hospital due to upper respiratory tract infection and severe headache for about 2 weeks; she did not have any underlying disease. A diagnostic lumbar puncture was performed in the emergency room to rule out meningitis. After several minutes, the patient complained of mild weakness in her bilateral lower limbs and hypoesthesia below T10. Neurological examination showed normal motor function in the bilateral upper limbs with hypoesthesia and $4+/ 5$ motor function in the bilateral lower limbs. Whole-spine magnetic resonance imaging (MRI) was performed immediately, which showed high intensity signal at T10-S1 in the T2-weighted sagittal and axial cut images, indicating an acute intradural hemorrhage (Fig. 1). The patient's hematologic tests were normal, and she was not taking any medications, including anticoagulants. We initiated high-dose steroid therapy immediately after diagnosis. However, the patient's neurological status rapidly deteriorated after 12 hours to a $1 / 5$ motor score in the right leg and $4 / 5$ in the left leg. We performed another diagnostic lumbar MRI and found no apparent increases in hematoma volume or spinal cord signal changes compared with the previous image.

\section{Surgical Procedure}

Decompressive laminectomy (L3 partial laminectomy and L4-5 total laminectomy) and 
hematoma removal were performed immediately. There was no hematoma in the epidural space, but the dura mater below the L3, 4, and 5 laminectomy was tense and bulging and had a bluish color. Durotomy was conducted longitudinally, the spinal subdural hematoma ( $\mathrm{SSdH})$ was visually identified, and evacuation was performed, and the arachnoid membrane was intact. Hematoma was found in the subarachnoid space and removed. The spinal cord and cauda equina were intact (Fig. 2). The surgery was completed after irrigation with normal saline. Postoperative cerebrospinal fluid (CSF) leakage was not observed.
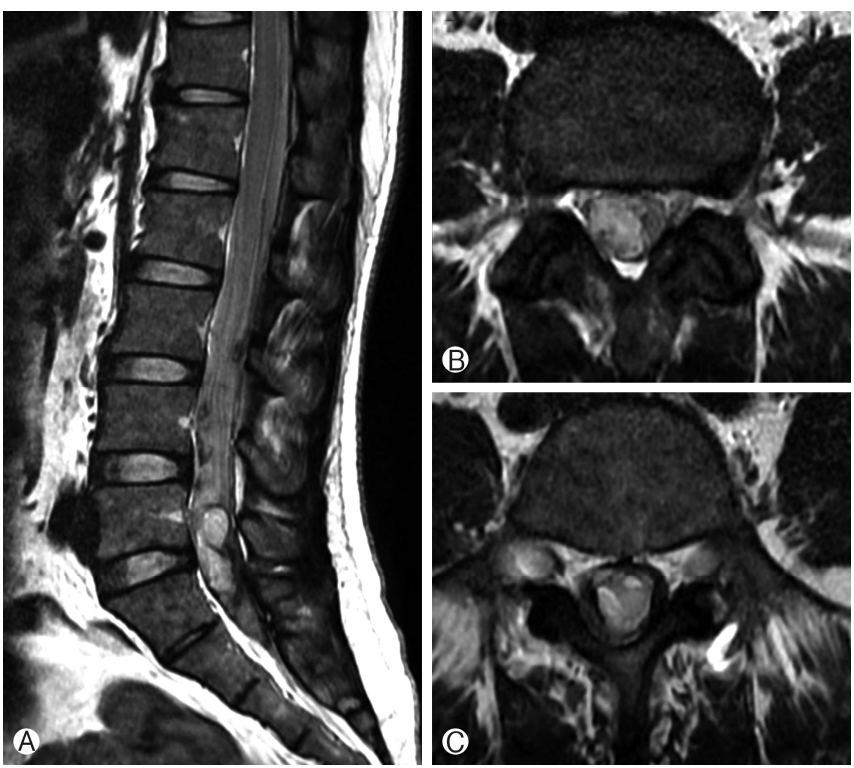

Fig. 1. (A) Sagittal T2-weighted magnetic resonance image showing high signal intensity lesions suggestive of intradural hemorrhage at L5 (B) and T10 (C).
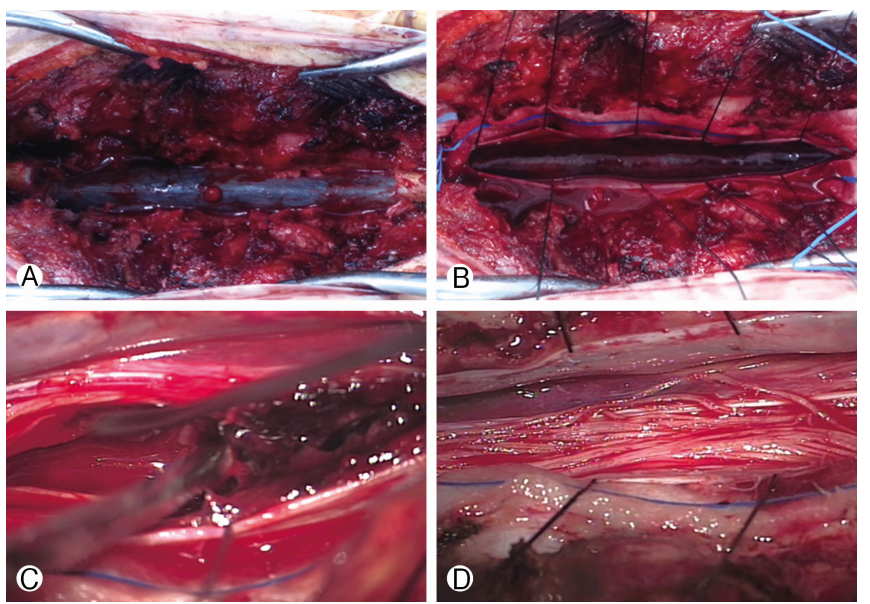

Fig. 2. (A) Intraoperative appearance of the dura mater after L3-5 decompressive laminectomy. (B) Intact arachnoid membrane and spinal subarachnoid hematoma after longitudinal durotomy. (C) Spinal subarachnoid hematoma after arachnoid membrane dissection. (D) Decompressed cauda equina after hematoma evacuation.

\section{Postoperative Course}

There was no evidence of remaining hematoma on a T2-weighted sagittal image obtained immediately after surgery (Fig. 3). The day after surgery the patient complained of a mild headache, and as such, brain computed tomography (CT) was performed; a subarachnoid hemorrhage in the posterior vertex sulci was observed (Fig. 4). However, we opted for conservative treatment because she had no other neurological symptoms. Brain CT performed at two weeks revealed that the subarachnoid hemorrhage had completely resolved, and right after surgery, the patient's motor function improved to $2 / 5$ in the right leg and $5 / 5$ in the left leg. Two days postoperative, she began rehabilitation, and 3 months thereafter, her motor function was $5 / 5$ in her lower limbs bilaterally, and her hypoesthesia had also resolved. The patient had no other neurologic deficits, and she was discharged.

\section{DISCUSSION}

SSH after lumbar puncture is very rare and is a critical compli-

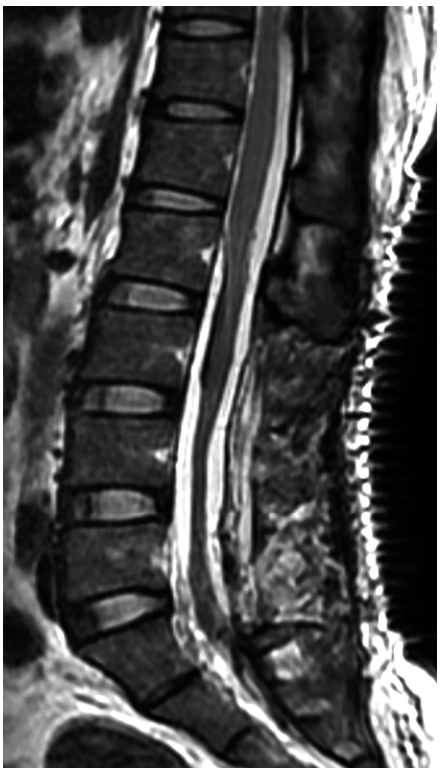

Fig. 3. Postoperative sagittal T2weighted image demonstrating resolution of the high signal intensity intradural hemorrhage and absence of L3-5 laminae.

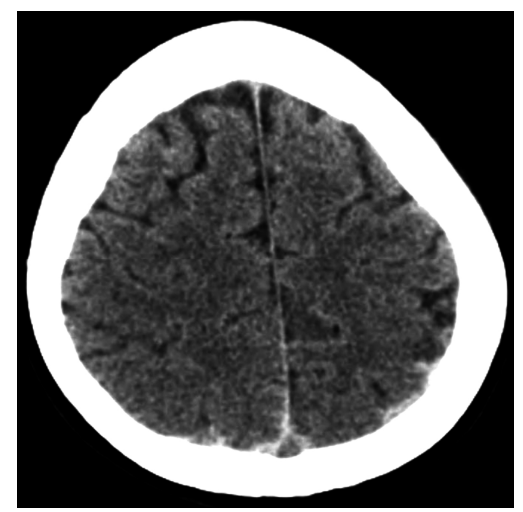

Fig. 4. Acute subarachnoid hemorrhage in the posterior sulci of the bilateral parietal lobes on nonenhanced axial brain computed tomography. 
cation that can cause permanent neurologic deficits ${ }^{1,3,9,16,18,20)}$. Plotkin et al. ${ }^{17)}$ introduced the concept of SSH in 1966. Most SSH cases are iatrogenic or are caused by trauma or a coagulation defect. According to Domenicucci et al. ${ }^{4)}, 40.5 \%$ of the $69 \mathrm{SSH}$ cases in their study were caused by coagulopathies, and the incidence of hematoma caused by lumber puncture was $44.9 \%$ and that due to traumatic injuries was $15.9 \%$. As in this study, 16 out of 69 patients (23\%) had simultaneous SSH and SSdH.

Hemorrhage may develop following lumbar puncture at the needling site or at a site slightly distal in addition to anywhere in the epidural, subdural, or subarachnoid spaces ${ }^{7,8)}$. SSH is a very rare lesion that is difficult to diagnose and frequently results in poor clinical outcomes compared with subdural or epidural hematomas ${ }^{7}$. A traumatic tap resulting in blood-stained CSF is most often due to puncture of the dorsal or ventral epidural venous plexuses and usually has no consequence. Masdeu et al. ${ }^{13)}$ suggested that SSH occurs when the radicular vessels are lacerated by traumatic lumbar puncture, and they confirmed this mechanism via autopsy. In SSH, as blood collects in the subarachnoid space, clots form, and as the clots press against the spinal cord and cauda equina, neurologic symptoms appear. In general, subarachnoid hemorrhage does not form blood clots because the blood is diluted with the flow of $\mathrm{CSF}^{6}$. For this reason, subarachnoid hemorrhage can be observed not only in the lesion but also at other levels on imaging. In the case in this report, we observed subarachnoid hemorrhage in the brain in addition in to the L4/5 lesion, where the lumbar puncture was conducted, and we also found a spinal intradural hemorrhage at T7. Damage to the blood vessel where the lumbar puncture was done resulted in a local hemorrhage that progressed to distant sites due to the CSF flow. If the hemorrhage from the damaged blood vessel is massive and the blood flow is fast, blood clots will form, and if the resulting hematoma causes compression of the spinal cord and cauda equina, neurologic symptoms will develop.

The most sensitive imaging available when the aforementioned symptoms appear is lumbar MRI ${ }^{15}$. Patients with SSH who have neurologic symptoms can be treated with surgical decompression and hematoma removal, and neurological recovery is related to the severity and speed of the preoperative deficit development and surgical decompression. Hematoma resolution and the neurologic impairment severity have the greatest impact on patient management and outcomes ${ }^{20)}$. In patients with rapidly progressing neurologic symptoms, early surgical intervention and evacuation are indicated, whereas in some patients with minimal symptoms, conservative treatment may play a role ${ }^{5)}$. Good results may be expected in patients with mild preoperative neurological deficits. Outcomes are usually poor in patients with SSH or severe preoperative deficits and in whom surgery has been delayed ${ }^{4,12}$.

Our patient had early neurological symptoms with only hypoesthesia; however, she developed paraplegia only 12 hours later. More interesting is that the lumbar MRI taken after 12 hours was no different from the previous study performed immediately after her hypoesthesia was noted. In our opinion, this delayed sudden paraplegia was due primarily to mechanical damage and cord compression associated with the mass effect of the hema- toma. As a result, we thought that edema and ischemic damage to the spinal cord had caused the neurological deficits ${ }^{2}$. However, this mechanism alone does not fully explain the delayed presentation of our patient's neurological symptoms. We believe that molecular pathophysiology including blood cytotoxicity, hypermetabolism, excitotoxicity, oxidative stress, and inflammation induced by spinal hemorrhage resulted in delayed damage to her spinal cord cells ${ }^{19)}$.

\section{CONCLUSION}

SSH is a rare complication of diagnostic lumbar puncture that may lead to disability if not properly treated in patients with neurologic deficits. Patients who complain of acute back pain or who demonstrate neurologic symptoms following lumbar puncture or similar spinal procedure should undergo MRI. If the diagnosis is confirmed to be SSH, surgical decompression of the spinal cord and cauda equina and hematoma evacuation must be done immediately to ensure neurologic recovery.

\section{CONFLICT OF INTEREST}

No potential conflict of interest relevant to this article was reported.

\section{REFERENCES}

1. Bong HJ, Kim JT, Jang KS, Chung DS, Park YS: Idiopathic, spontaneous thoracic subarachnoid hematoma. Korean J Spine 4:72-75, 2007

2. Dampeer RA: Spontaneous spinal subdural hematoma: case study. Am J Crit Care 19:191-193, 2010

3. Domenicucci M, Ramieri A, Ciappetta P, Delfini R: Nontraumatic acute spinal subdural hematoma: report of five cases and review of the literature. J Neurosurg 91(1 Suppl):65-73, 1999

4. Domenicucci M, Ramieri A, Paolini S, Russo N, Occhiogrosso G, Di Biasi C, et al: Spinal subarachnoid hematomas: our experience and literature review. Acta Neurochir (Wien) 147:741-750, 2005

5. Egede LE, Moses H, Wang H: Spinal subdural hematoma: a rare complication of lumbar puncture. Case report and review of the literature. Md Med J 48:15-17, 1999

6. Gaitzsch J, Berney J: Spinal subarachnoid hematoma of spontaneous origin and complicating anticoagulation. Report of four cases and review of the literature. Surg Neurol 21:534-538, 1984

7. Gerancher JC, Waterer R, Middleton J: Transient paraparesis after postdural puncture spinal hematoma in a patient receiving ketorolac. Anesthesiology 86:490-494, 1997

8. Goyal A, Dua R, Singh D, Kumar S: Spinal subarachnoid haematoma following lumbar puncture. Neurol India 47:339-340, 1999

9. Horlocker TT, Wedel DJ, Schroeder DR, Rose SH, Elliott BA, McGregor DG, et al: Preoperative antiplatelet therapy does not increase the risk of spinal hematoma associated with regional anesthesia. Anesth Analg 80:303-309, 1995

10. Katoh H, Manabe K, Shimizu A, Shima K, Chigasaki H, Tsuchiya $\mathrm{K}$ : A case of traumatic spinal subarachnoid hematoma causing compression of the cauda equina. No Shinkei Geka 20:1119-1123, 1992 
11. Lawton MT, Porter RW, Heiserman JE, Jacobowitz R, Sonntag VK, Dickman CA: Surgical management of spinal epidural hematoma: relationship between surgical timing and neurological outcome. J Neurosurg 83:1-7, 1995

12. Likar R, Mathiaschitz K, Spendel M, Krumpholz R, Martin E: Acute spinal subdural hematoma after attempted spinal anesthesia. Anaesthesist 45:66-69, 1996

13. Masdeu JC, Breuer AC, Schoene WC: Spinal subarachnoid hematomas: clue to a source of bleeding in traumatic lumbar puncture. Neurology 29:872-876, 1979

14. Matsumura A, Namikawa T, Hashimoto R, Okamoto T, Yanagida I, Hoshi $\mathrm{M}$, et al: Clinical management for spontaneous spinal epidural hematoma: diagnosis and treatment. Spine J 8:534-537, 2008

15. Nolli M, Crispino M, Nicosia F, Borghi B, Montone N: Diagnosis and therapy of intrathecal bleeding. Minerva Anestesiol 67(9 Suppl 1):82-91, 2001
16. Owens EL, Kasten GW, Hessel EA 2nd: Spinal subarachnoid hematoma after lumbar puncture and heparinization: a case report, review of the literature, and discussion of anesthetic implications. Anesth Analg 65:1201-1207, 1986

17. Plotkin R, Ronthal M, Froman C: Spontaneous spinal subarachnoid haemorrhage. Report of 3 cases. J Neurosurg 25:443-446, 1966

18. Robin C, Salzmann M, Loquineau-Wei TH, Raynaud A, Duthel R: Spontaneous spinal epidural hematoma: 2 cases. Rev Neurol (Paris) 152:139-142, 1996

19. Rosenberg GA: Matrix metalloproteinases in neuroinflammation. Glia 39:279-291, 2002

20. Segabinazzi D, Brescianini BC, Schneider FG, Mendes FF: Conservative treatment of hematoma after spinal anesthesia: case report and literature review. Rev Bras Anestesiol 57:188-194, 2007 